\title{
O PEDAGOGO E AS NOVAS TECNOLOGIAS
}

\section{Elisa Daniele de Andradex ${ }^{1}$ Jaqueline Lesinhovski Talamini, Marcia Patricia kuligovski}

${ }^{1}$ Pedagoga, Diretora do Departamento de Tecnologia Educacional da Secretaria Municipal de Educação, professora do Ensino Fundamental no município de Araucária/PR

${ }^{2}$ Pedagoga, Mestre em Educação, professora do Ensino Fundamental no município de Araucária/PR

${ }^{3}$ Pedagoga, professora do Ensino Fundamental no município de Araucária/PR elisa.dani@ig.com.br, jaquelinetalamini8@gmail.com, patikulig@gmail.com

\begin{abstract}
This paper discusses the use of new technologies as resources in the teaching and learning, focusing on the importance of teacher mediation to integrate these new tools in education. The research was conducted with the teachers participating in the course "The teacher and the Information and Communication technologies: in search of a real connection" performed in a bimodal fashion, in Araucaria. The course met a group of twenty-nine teachers in public schools fifteen. We tried to get up during the research data on the vision of the professional educator related to technology in education and the need for continuing education for the integration of new technologies in teaching and learning.
\end{abstract}

Resumo. O presente trabalho aborda a utilização das novas tecnologias como recursos no processo de ensino e aprendizagem, com enfoque na importância da mediação do pedagogo para a integração dessas novas ferramentas na educação. $A$ pesquisa foi realizada junto aos pedagogos participantes do curso "O pedagogo e as tecnologias de Informação e Comunicação: em busca de uma conexão real" realizado na forma bimodal, no município de Araucária. O curso atendia um grupo de vinte e nove pedagogos, de quinze escolas municipais. Buscou-se no decorrer da pesquisa levantar dados sobre a visão do profissional pedagogo relacionado às tecnologias na educação e a necessidade da formação continuada para a integração das novas tecnologias no processo de ensino e aprendizagem.

\section{Introdução}

O relato a seguir teve como objetivo avaliar a contribuição da formação continuada dos pedagogos para o uso das tecnologias da informação e comunicação no contexto escolar. 
Primeiramente investigou-se o conceito prévio de tecnologia trazido pelo grupo de pedagogos, antes da formação. Ao término da formação foram analisadas as opiniões dos mesmos a respeito da importância dos recursos tecnológicos na educação, bem como as possibilidades e limitações destes profissionais frente aos recursos tecnológicos.

\section{As Tecnologias Educacionais e a Formação Continuada}

As novas tecnologias presentes na sociedade podem contribuir significativamente no processo de ensino e de aprendizagem e alterar a maneira de ensinar e aprender. Brito e Purificação (2006, p. 19) classificam as tecnologias em três grupos:

- Tecnologias Físicas: compreendem os objetos, instrumentos físicos, como: livro, computador, telefone, lápis...

- Tecnologias Organizadoras: são tecnologias que permitem organizar a forma com que nos relacionamos com o mundo, como, na escola o Projeto Político Pedagógico.

- Tecnologias Simbólicas: são tecnologias relacionadas a forma de comunicação entre as pessoas, como a linguagem oral, linguagem escrita, libras, Braille...

Ainda de acordo com Brito e Purificação (2006) as tecnologias estão fortemente interligadas e são interdependentes. Uma determinada tecnologia perpassa dois ou mais dos grupos quando classificada. Os profissionais da educação precisam buscar a integração desses recursos na prática pedagógica, para isso a formação continuada é fundamental.

Programas do governo federal como o PROINFO (Programa Nacional de Informática na Educação) e o UCA (Um Computador por Aluno), além de iniciativas da administração estadual e municipal, tem implantado recursos tecnológicos nas escolas.

O investimento na aquisição e manutenção de equipamentos é importante para a inclusão digital, mas é essencial o investimento na formação continuada do professor, responsável pela mediação no processo de ensino e aprendizagem, para que se efetive a integração das novas tecnologias na educação, para que não se reproduza uma velha prática pedagógica com o uso de novas ferramentas, de maneira fragmentada $\mathrm{e}$ desarticulada dos conteúdos curriculares.

Em boa parte das escolas as novas tecnologias já estão incluídas, disponíveis no ambiente escolar, mas não há real integração ou exploração de suas potencialidades na prática pedagógica.

A formação do professor tem que contemplar o uso das tecnologias educacionais para que ocorram mudanças na escola. Formação esta que permita a reflexão na ação docente, buscando não somente a apropriação da técnica, mas, principalmente, que 
articule o uso das novas tecnologias de maneira pedagógica. E nesta perspectiva o papel do pedagogo é fundamental.

O pedagogo é o profissional responsável pela organização e coordenação do trabalho pedagógico desenvolvido nas escolas. Dessa forma, ele organiza momentos de formação continuada, promovendo subsídios teóricos e práticos, que objetivam qualificar o ensino e a aprendizagem, desde o planejamento até a avaliação de todos os processos pedagógicos.

De acordo com Vasconcellos (2004), entende-se o pedagogo como autoridade educativa nas dimensões: profissional, intelectual, ética e humana, que trabalha em prol de uma educação que promove a formação humana e fortalece o sujeito confrontado com a necessidade de aprender, na busca de conhecimentos que o façam entender a sua história, a sua cultura e as relações sociais que esse sujeito estabelece, ou seja, um sujeito que age no e sobre o mundo que se produz a si mesmo e que é produzido através da educação.

Portanto, o pedagogo é gestor, que de acordo com Ferreira (2001) é aquele que coordena ou direciona a prática que concretiza uma linha de ação, um plano de mudanças a serem efetivadas.

O trabalho do pedagogo é essencial para subsidiar e articular a integração das novas tecnologias no processo de ensino e de aprendizagem, auxiliando os professores nos momentos de planejamento, pesquisa e elaboração de atividades, escrita e análise de projetos escolares, promovendo a inclusão digital e desenvolvendo novas estratégias de trabalho com os recursos tecnológicos e também aprimorando as práticas que já estão sendo desenvolvidas no interior das escolas.

\section{A Tecnologia Educacional em Araucária}

A Prefeitura do Município de Araucária, Estado do Paraná, implantou um Projeto de Tecnologia Educacional no ano de 2004. No início do Projeto foram instalados laboratórios em doze escolas da rede municipal, dois laboratórios de mesas pedagógicas e um laboratório itinerante com vinte notebooks que percorriam as demais escolas da rede onde não havia laboratórios fixos.

Em 2009, na Secretaria Municipal de Educação, foi criado o Departamento de Tecnologia Educacional - DTED e homologado o Núcleo de Tecnologia Municipal NTM junto ao Ministério da Educação - MEC, resultando em convênio junto ao Programa Nacional de Informática da Educação - PROINFO. Como resultado desse convênio foram implantados no município novos laboratórios de informática. Atualmente, trinta e seis das quarenta e duas escolas do município, já possuem laboratórios informática.

Ainda em 2009, teve início o planejamento para a implantação do Projeto UCAA - Um Computador por Aluno em Araucária. Esse Projeto pretende até o final de 
2012 disponibilizar um computador por aluno, na modalidade 1:1 por turno, em toda a rede municipal, bem como disponibilizar um computador para cada professor da rede. Atualmente das 37 escolas municipais, 24 já tem o projeto implantado.

O Departamento de Tecnologia Educacional também é responsável pela estruturação, planejamento e desenvolvimento da formação continuada dos professores em tecnologias educacionais. A formação atende os professores, pedagogos e diretores dos estabelecimentos de ensino municipais.

Em 2010, tendo em vista a importante função do pedagogo como articulador da integração das novas tecnologias na educação, foi inserido no Plano de Ação do Departamento um curso bimodal (aulas presenciais e a distância) com o objetivo de proporcionar formação continuada aos pedagogos para o uso das tecnologias da informação e comunicação no contexto escolar.

Para o desenvolvimento do curso foi utilizado o ambiente virtual de aprendizagem Moodle, disponibilizado pela Universidade Federal do Paraná. A partir deste ambiente que se estabelecia a comunicação entre a professora mediadora e os pedagogos participantes, bem como as atividades e avaliações a serem realizadas.

Os pedagogos participantes do curso atuavam nas 15 primeiras escolas da rede municipal que receberam o Projeto UCAA - Um Computador por Aluno em Araucária em 2010. Pretendeu-se com o curso possibilitar ao pedagogo, conhecimento técnico e, especialmente, pedagógico para auxiliar o professor em seu planejamento nas atividades que envolvam o uso do computador como recurso, compreendendo o que é, para que serve e os benefícios que o mesmo traz para o processo de ensino e aprendizagem.

\section{Público alvo}

O público alvo da pesquisa compreendeu um grupo de 29 pedagogos do Curso de Extensão Universitária "O pedagogo e as tecnologias de informação e comunicação: em busca de uma conexão real", ofertado pela Secretaria Municipal de Educação de Araucária, através do Departamento de Tecnologia Educacional, em parceria com a Universidade Federal do Paraná.

\section{Metodologia da pesquisa}

Para a coleta de dados utilizou-se o ambiente virtual de aprendizagem do curso e foram aplicados também questionários aos cursistas. Foi uma pesquisa de cunho qualitativo e quantitativo. Os dados coletados foram analisados e sistematizados em quadros e na forma de texto.

\section{Análise dos dados}

No início do curso utilizando o ambiente virtual, os pedagogos foram questionados sobre qual conceito tinham sobre tecnologia. Algumas colocações dos pedagogos 
deixaram evidente que o conceito de tecnologia está fortemente relacionado unicamente com o objeto em si, o instrumento resultante de um processo de produção, ou seja, uma tecnologia física.
"Tecnologia é tudo o que utilizamos para otimizar nosso tempo: celular, computador, microondas, enfim, todos os instrumentos que nos auxiliam em nosso dia a dia e em nosso trabalho". Pedagogo A

"Tecnologia é tudo o que utilizamos para agilizar e modernizar o nosso trabalho." Pedagogo B

Alguns pedagogos colocaram sua conceituação de tecnologia de forma mais abrangente, não se limitando ao produto tecnológico, mas perpassando pelo conhecimento necessário para chegar a esse produto.

"A tecnologia está ligada ao conhecimento técnico e científico, as ferramentas criadas para auxiliar as pessoas diariamente (escrita, transportes, vídeo, internet, eletrônicos...) na resolução de problemas". Pedagogo $\mathrm{H}$

Também percebeu-se no conceito de tecnologia apresentado pelos pedagogos, uma relação entre a tecnologia e as mudanças sociais e, consequentemente, sua influência na vida das pessoas.
"A tecnologia causa mudança nas pessoas, traz novas formas de ler e escrever, portanto, de pensar e agir. Nos ajuda, nos comple- ta, nos amplia. Traz novas mudanças sociais e culturais. A tec- nologia é um recurso, é uma ferramenta. Com o uso correto pode transformar-se num aliado, estando a serviço do homem e da educação". Pedagogo K

Analisando as colocações verificou-se que o grupo de pedagogos, em sua maioria, tinha como conceito de tecnologia inicialmente a tecnologia física o que reforçava a necessidade de discussão na formação sobre o tema, para ampliar a visão sobre tecnologia e sua relação com a educação.

Nos encontros presenciais foram realizadas discussões, trabalhos em grupos, seminários e atividades utilizando o computador. Os conteúdos abordados no curso foram: conceito de tecnologia educacional; função do pedagogo diante dos avanços tecnológicos; relação da tecnologia no contexto atual; utilização de recursos tecnológicos no ensino; internet; tecnologias assistivas e recursos de acessibilidade.

Após o curso de extensão foi aplicado um questionário aos pedagogos. $\mathrm{O}$ primeiro questionamento buscou evidenciar a opinião dos pedagogos a respeito da importância da utilização das novas tecnologias na prática pedagógica. Todos os 
pedagogos que responderam a pesquisa concordam que as novas tecnologias são um importante diferencial no processo de ensino e aprendizagem. (QUADRO 1).

\section{QUADRO 2 - IMPORTÂNCIA DA UTILIZAÇÃO DAS NOVAS TECNOLOGIAS NO PROCESSO PEDAGÓGICO.}

\begin{tabular}{|c|c|}
\hline Pedagogo & Importância da utilização das novas tecnologias no processo pedagógico: \\
\hline Pedagogo 1 & "Sim. A utilização das novas tecnologias estão para enriquecer o trabalho pedagógico". \\
\hline Pedagogo 2 & $\begin{array}{l}\text { "Muito importante, uma vez que a tecnologia ocupa um espaço cada vez maior na } \\
\text { sociedade, a escola não pode desconsiderá-la e sim utiliza-la como aliada no processo } \\
\text { de aprendizagem". }\end{array}$ \\
\hline Pedagogo 3 & $\begin{array}{l}\text { "Considero. As novas tecnologias possibilitam inovar o processo de ensino e } \\
\text { aprendizagem. É através de seu uso que os educadores e educandos podem, também, } \\
\text { socializar o conhecimento". }\end{array}$ \\
\hline Pedagogo 4 & "Sim, é uma ferramenta de grande importância no fazer pedagógico do professor ..." \\
\hline Pedagogo 5 & $\begin{array}{l}\text { "Sim. A tecnologia faz parte da modernidade. É necessário que todos estejamos } \\
\text { inseridos neste processo. A escola pode aproveitar este recurso que está disponível, } \\
\text { enriquecendo o trabalho pedagógico visando a qualidade da educação". }\end{array}$ \\
\hline Pedagogo 6 & $\begin{array}{l}\text { "Sim. Ajuda na compreensão de determinados conteúdos, incentiva os alunos, } \\
\text { enriquece as aulas". }\end{array}$ \\
\hline Pedagogo 7 & "Sim. Ajuda muito na aprendizagem dos alunos". \\
\hline Pedagogo 8 & $\begin{array}{l}\text { "Sim. Contribuindo, principalmente, para a motivação, curiosidade, interesse por parte } \\
\text { de todos os envolvidos neste processo". }\end{array}$ \\
\hline Pedagogo 9 & $\begin{array}{l}\text { "Sim, com a velocidade que a tecnologia está entrando em nosso cotidiano não tem } \\
\text { como ficar alheio". }\end{array}$ \\
\hline Pedagogo 10 & $\begin{array}{l}\text { "Sim, pois os alunos, mesmo os que não possuem as ferramentas em casa, estão } \\
\text { conectados as novas tecnologias e formas de conhecimento, então a necessidade de nos } \\
\text { inserirmos nesse processo". }\end{array}$ \\
\hline Pedagogo 11 & "Sim, pois fazem parte do processo de humanização". \\
\hline Pedagogo 12 & $\begin{array}{l}\text { "Sim, pois a educação é um processo dinâmico e contínuo. Ela bem utilizada pode } \\
\text { tornar esse processo criativo e inovador". }\end{array}$ \\
\hline Pedagogo 13 & $\begin{array}{l}\text { "Por acreditar que as novas tecnologias contribuem bis aspectos relacionados acima e } \\
\text { muitos outros, possibilitando aulas mais atrativas e dinâmicas e levando o professor a } \\
\text { buscar conhecimento e sair da zona de conforto". }\end{array}$ \\
\hline
\end{tabular}

Como mostram as respostas, os pedagogos apontam como um dos principais motivos para a integração das novas tecnologias no processo pedagógico, o fato desses recursos estarem presentes em diversas áreas da sociedade e a importância da escola preparar os alunos para a sociedade da informação, além de acompanhar e aproveitar a motivação dos avanços tecnológicos para não ficar aquém dessa evolução.

Embora todos os pedagogos considerem a integração das novas tecnologias importante, ressaltam vários motivos que podem impedir que se efetive a articulação dos recursos tecnológicos no ambiente escolar. (QUADRO 2): 


\begin{tabular}{|c|c|}
\hline Pedagogo & $\begin{array}{l}\text { Principais motivos que impedem que se efetive a integração das novas } \\
\text { tecnologias no ambiente escolar: }\end{array}$ \\
\hline Pedagogo 1 & "A própria barreira que o profissional tem perante o manuseio das tecnologias". \\
\hline Pedagogo 2 & $\begin{array}{l}\text { "A insegurança dos profissionais quanto ao seu uso. A estrutura física (instalações } \\
\text { elétricas, espaço adequado)". }\end{array}$ \\
\hline Pedagogo 3 & $\begin{array}{l}\text { "Resistência de alguns profissionais em relação a mudança, falta de formação, } \\
\text { estrutura que possibilite o uso". }\end{array}$ \\
\hline Pedagogo 4 & $\begin{array}{l}\text { "Eu quero que este fator não interfira, mas o impedimento que poderia ocorrer } \\
\text { seria o medo pelo novo, a insegurança". }\end{array}$ \\
\hline Pedagogo 5 & "A disponibilidade para aprender, sair da acomodação e reinventar". \\
\hline Pedagogo 6 & "A falta de ligarem o laboratório em nossa escola". \\
\hline Pedagogo 7 & "O medo dos profissionais". \\
\hline Pedagogo 8 & $\begin{array}{l}\text { "Muitas vezes pela desorganização da rotina escolar, na qual os profissionais } \\
\text { ficam imersos e em outros momentos o suporte técnico de fundamental } \\
\text { importância". }\end{array}$ \\
\hline Pedagogo 9 & "Acredito que existe resistência em função de que as novidades sempre assustam". \\
\hline Pedagogo 10 & $\begin{array}{l}\text { "Medo do desconhecido, acomodação, incertezas. Contudo, acredito que é fase, } \\
\text { pois com a velocidade que está sendo inserido as tecnologias em nossas vidas ou } \\
\text { você absorve ou será absorvido por ela". }\end{array}$ \\
\hline Pedagogo 11 & $\begin{array}{l}\text { "Falta formação continuada, falta de compromisso ético no respeito ao direito do } \\
\text { aluno". }\end{array}$ \\
\hline Pedagogo 12 & $\begin{array}{l}\text { "O medo do novo. A resistência cristalizada do 'não quero me incomodar' de } \\
\text { alguns profissionais, porém não podemos nos deter nestes, mas sim nos outros } \\
\text { abertos para esse novo olhar". }\end{array}$ \\
\hline Pedagogo 13 & $\begin{array}{l}\text { "Condições físicas, de infraestrutura das escolas; Resistência ao novo, } \\
\text { comodismo; Insegurança, falta de domínio técnico dos profissionais". }\end{array}$ \\
\hline
\end{tabular}

As respostas evidenciam a insegurança do professor, a falta de interesse e o medo em deixar sua zona de conforto e buscar aperfeiçoamento. Alguns pedagogos destacam também a falta de infraestrutura adequada como um impedimento para a utilização desses novos recursos na educação.

$\mathrm{Na}$ sequência os pedagogos foram questionados sobre a importância da formação continuada para a integração das novas tecnologias na educação e na sistematização (QUADRO 3), todos consideram de suma importância o investimento na formação continuada. 


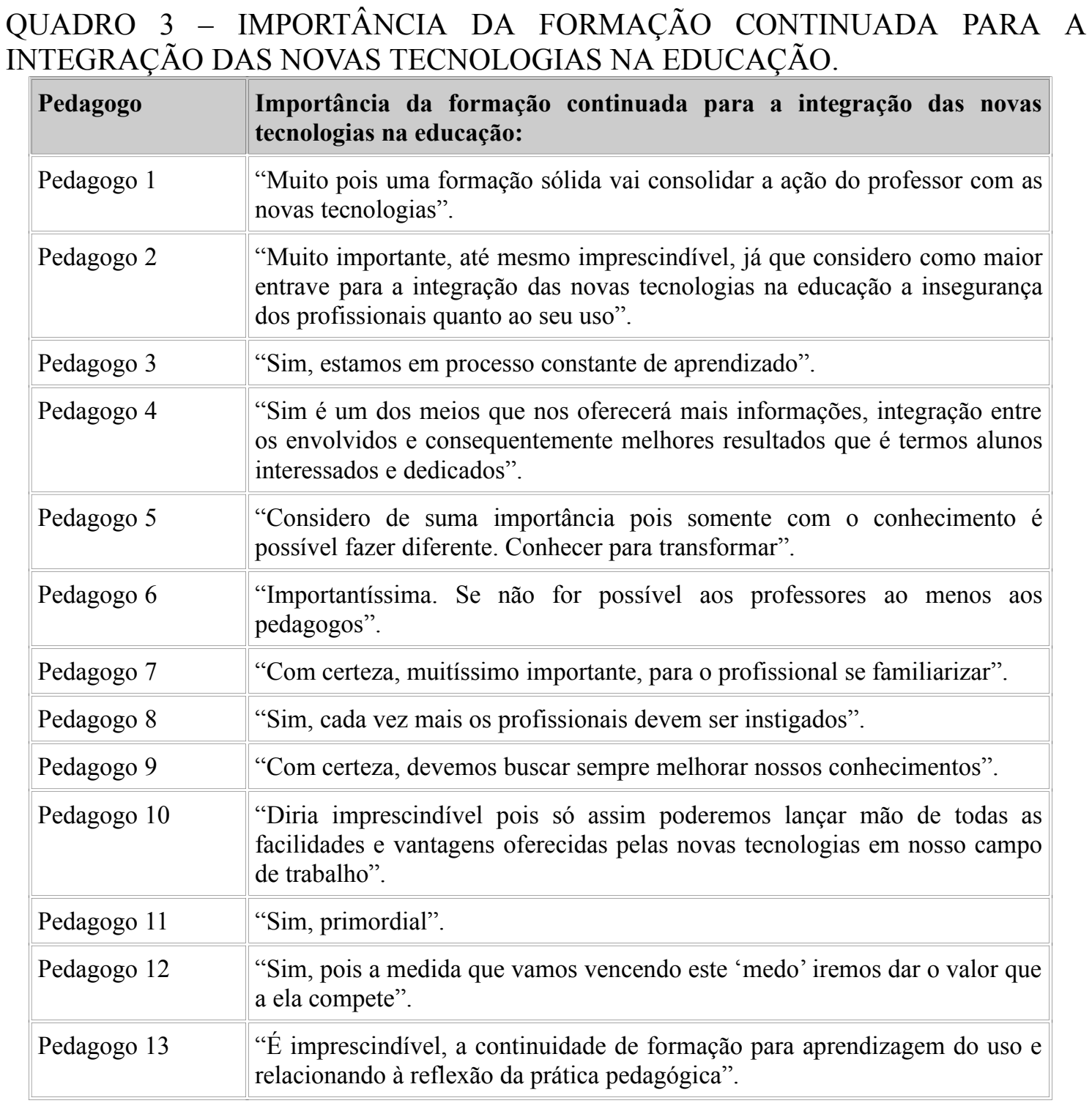

$\mathrm{Na}$ formação inicial de muitos professores não foi contemplada uma disciplina relacionada às tecnologias educacionais, o que dificulta ainda mais a utilização dos recursos tecnológicas na prática pedagógica.

A formação continuada pode contribuir, como citado pelos pedagogos, para superar as dificuldades encontradas, dando subsídios para os professores se apropriarem de conhecimentos sobre as ferramentas e introduzir esses recursos em suas aulas.

Quando questionados sobre as contribuições do curso de extensão para a reflexão sobre o uso das tecnologias na escola, todos os pedagogos apontam que esse curso contribuiu para pensar o uso dos recursos tecnológicos como ferramentas que podem tornar as aulas mais dinâmicas e atrativas aos alunos. 


\section{QUADRO 4 - O CURSO REALIZADO MUDOU SUA MANEIRA DE PENSAR AS TECNOLOGIAS NA ESCOLA.}

\begin{tabular}{|c|c|}
\hline Pedagogo & O curso realizado mudou sua maneira de pensar as tecnologias na escola: \\
\hline Pedagogo 1 & $\begin{array}{l}\text { "Sim. Não podemos ignorar que temos a presença da tecnologia em nosso dia a } \\
\text { dia; o curso de formação serviu para enriquecer o conhecimento que tinha } \\
\text { sobre a tecnologia na escola". }\end{array}$ \\
\hline Pedagogo 2 & $\begin{array}{l}\text { "Sim. Me fez perceber que este é um caminho sem retorno, que precisamos } \\
\text { estudar e nos inteirar deste assunto para que o uso das tecnologias na escola } \\
\text { contribuam com o processo de ensino e aprendizagem". }\end{array}$ \\
\hline Pedagogo 3 & $\begin{array}{l}\text { "Sim. Ampliou minha visão sobre como é possível fazer uso de diferentes } \\
\text { tecnologias para despertar o interesse do aluno e contribuir na ação } \\
\text { pedagógica". }\end{array}$ \\
\hline Pedagogo 4 & $\begin{array}{l}\text { "Sim, com certeza. Através das informações recebidas nas formações } \\
\text { continuadas e mesmo na troca de sugestões de endereços eletrônicos, } \\
\text { pesquisas... que são trocados e/ou sugeridos entre colegas de trabalho". }\end{array}$ \\
\hline Pedagogo 5 & $\begin{array}{l}\text { "Mudou no sentido de perceber que está ao alcance de todos que desejem, isto } \\
\text { é uma novidade. Estamos todos mais ou menos no mesmo nível de } \\
\text { conhecimento e podemos contar um com outro. Também há pessoas mais } \\
\text { experientes dispostas a contribuir". }\end{array}$ \\
\hline Pedagogo 6 & $\begin{array}{l}\text { "Sim, pois acrescentou mais conhecimentos, informações, enfim clareou } \\
\text { muitas dúvidas que existiam também". }\end{array}$ \\
\hline Pedagogo 7 & $\begin{array}{l}\text { "Sim. Os recursos são muitos e podemos abranger infinitas áreas e fazer } \\
\text { relações constantes (passado, futuro, presente). O entendimento se transforma } \\
\text { e nos transforma". }\end{array}$ \\
\hline Pedagogo 8 & $\begin{array}{l}\text { "Com certeza, pois me instigou a buscar leituras sobre este assunto, a discutir } \\
\text { com os docentes e mediar as atividades pedagógicas". }\end{array}$ \\
\hline Pedagogo 9 & $\begin{array}{l}\text { "Sim. Acredito que as reflexões realizadas contribuíram para a inovação das } \\
\text { ações no ambiente escolar". }\end{array}$ \\
\hline Pedagogo 10 & $\begin{array}{l}\text { "Certamente que sim, sei que posso enriquecer uma aula, reunião, fazendo uso } \\
\text { do conhecimento adquirido, além de me possibilitar condições de ir em buscar } \\
\text { de novos saberes". }\end{array}$ \\
\hline Pedagogo 11 & "Sim, sempre agrega referência para trabalhar com os professores". \\
\hline Pedagogo 12 & $\begin{array}{l}\text { "Sim. A dar-lhe mais importância na sua utilização e mobilizar os demais } \\
\text { profissionais em como ela pode agilizar e facilitar o nosso trabalho". }\end{array}$ \\
\hline Pedagogo 13 & $\begin{array}{l}\text { "Contribuiu muito na reflexão aprofundando conhecimentos que auxiliarão no } \\
\text { nosso trabalho e acompanhamento do professor". }\end{array}$ \\
\hline
\end{tabular}

Por meio dos comentários os pedagogos consideraram o curso de extensão uma importante formação para articular as tecnologias ao processo de ensino-aprendizagem, propiciando uma análise mais ampla das possibilidades que os recursos tecnológicos apresentam para as aulas.

As respostas dos pedagogos expressam a visão de que as novas tecnologias podem contribuir significativamente no processo de ensino e aprendizagem e que a formação continuada é o grande diferencial para que haja uma real integração desses recursos a prática pedagógica. 


\section{Considerações}

O uso de novas tecnologias vem adquirindo cada vez mais relevância no cenário educacional. Sua utilização como recurso no processo de ensino-aprendizagem e sua ação no meio social vem aumentando rapidamente. Nesse sentido, a educação vem passando por mudanças estruturais e funcionais frente a essas novas tecnologias.

O ambiente escolar precisa e deve usar toda a tecnologia disponível para desenvolver seu trabalho pedagógico usufruindo, assim, dos benefícios que ela pode trazer para o desenvolvimento dos educandos, oportunizando a eles uma educação voltada para sua realidade e dando condições de participar ativamente na sociedade contemporânea.

Durante a pesquisa os pedagogos apontaram a formação continuada para o uso das novas tecnologias na educação essencial para que se efetive a integração e o professor possa ter autonomia na utilização dessas ferramentas em sua prática pedagógica.

Esses profissionais entendem que as novas tecnologias contribuem para a aprendizagem, porém alguns fatores, especialmente, a insegurança e a falta de preparo para explorar algo desconhecido, podem dificultar sua efetiva utilização.

\section{REFERÊNCIAS}

ARAUCÁRIA. Secretaria Municipal de Educação. Departamento de Tecnologia Educacional. Plano de Formação 2010. SMED: Araucária, 2010.

BRITO, Glaucia da Silva; PURIFICAÇÃO, Ivonélia da. Educação e novas tecnologias: um re-pensar. Curitiba: Ibpex, 2006.

FERREIRA, N. (Org.) Gestão democrática da educação: atuais tendências, novos desafios. 3. ed. Cortez: São Paulo, 2001.

KALINKE, Marco Aurélio. Para não ser um professor do século passado. Curitiba: Chain, 1999.

VASCONCELLOS, C. Coordenação do trabalho pedagógico: do projeto político-pedagógico ao cotidiano da sala de aula. São Paulo: Libertad, 2004. 